\title{
El desarrollo psicomotor y la calidad de la atención temprana
}

\author{
Lourdes Huiracocha T. ${ }^{1}$, Gladys Robalino I. ${ }^{1}$, Miriam S. Huiracocha T. ${ }^{1}$, Jorge L. García A. ${ }^{1}$, \\ Carmen G. Pazán T. ${ }^{1}$, Aydeé Angulo ${ }^{1}$, Carlos A. Almeida D. ${ }^{2}$ \\ ${ }^{1}$ Facultad de Ciencias Médicas, Universidad de Cuenca, Av. 12 de Abril s/n, Cuenca, Ecuador. \\ ${ }^{2}$ Departamento de Protección Integral, Instituto de la Niñez y la Familia, Av. Remigio Crespo, \\ Cuenca, Ecuador.
}

Autor para correspondencia: lourdes.huiracocha@ucuenca.edu.ec

Fecha de recepción: 15 de abril 2012 - Fecha de aceptación: 18 de diciembre 2012

\begin{abstract}
RESUMEN
Este estudio transversal determinó la relación entre alerta del desarrollo psicomotor de los niños de 0 a 5 años de edad y la calidad individual de atención temprana en los Centros de Desarrollo Infantil públicos y privados de la Municipalidad de Cuenca de Agosto a Diciembre del 2009 en las dimensiones de proceso de atención, preparación profesional, infraestructura, participación familiar. Mediante muestra clúster de 166 centros se sorteó a 30 de los cuales se aleatorizó la muestra de los niños calculando con el 5\% de frecuencia de retraso del desarrollo, el 3\% de inferencia, el 95\% de confianza y el $10 \%$ de pérdidas $(\mathrm{N}=8145, \mathrm{n}=447)$, se excluyeron a los niños con diagnóstico previo de discapacidad. La recolección fue a través de la observación, a los niños se les evaluó el desarrollo psicomotor con la escala abreviada de Nelson-Ortiz (1999). El 26,7\% de los centros fueron públicos, el 23,3\% no tuvo el registro legal, el $10 \%$ integró a niños con discapacidad, el $20 \%$ presentó calidad muy buena, el $3,3 \%$ buena y el $76,7 \%$ mala. De los niños del estudio el $26 \%$ no fueron evaluados en sus centros, el 72,7\% tuvo profesoras sin título profesional. La prevalencia de los niños con alerta en el desarrollo fue del 10,5\% (IC 95\%: 7,8 a 13,7\%), de estos el 79,6\% no tuvo el registro de su detección y el 93,9\% no fue derivado para el diagnóstico ni intervención ni tuvo adaptaciones curriculares. Existió relación significativa $(\mathrm{p}<0,05)$ entre la calidad de atención temprana y la alerta en el desarrollo de los niños. Estos resultados deben llevar a un plan de mejoramiento.
\end{abstract}

Palabras clave: Atención, jardines infantiles, indicadores de calidad, vigilante, preescolar, desarrollo psicomotor.

\begin{abstract}
A transversal study was conducted to determine the relation between alert psychomotor development of children, 0 to 5 years old, and the quality of early attention of public and private Child Development Centers in the Municipality of Cuenca. The survey was conducted from August to December 2009 and consisted in measuring the attention process, the professional level of caretakers, available infrastructure, and family involvement. Using a simplified cluster sampling approach, 30 centers were randomly selected out of 166 centers. The composition of the sample of children was based on the following considerations: $5 \%$ delay in development, $3 \%$ error of inference, $95 \%$ confidence interval and $10 \%$ loss, resulting in a sample of 447 (n) out of a total study population of $8145(\mathrm{~N})$ children excluding the children with previous diagnosis of disability. To test the children's delay in development the scale of Nelson-Ortiz (1999) was used. 23,3\% of the centers were public, 23,3\% had no legal registration, $10 \%$ of the centers were serving children with disability, $20 \%$ were rated as having very good quality, $3,3 \%$ good and $76,7 \%$ poor. Of the children surveyed $26 \%$ were not evaluated in their respective centers, $72,7 \%$ had teachers without professional qualification. The prevalence of children with alert development was 10,5\% (IC 95\%: 7,8 to13,7\%), of these $79,6 \%$ had no record of detection and 93,9\% were not referred for diagnosis or intervention or curricular adaptations. A significant relationship $(\mathrm{p}<0,05)$ between the quality of early attention and the alert development of children was found.
\end{abstract}


Keywords: Attention, child day-care center, quality indicators, caretaker, preschool child, psychomotor development.

\section{INTRODUCCIÓN}

Los niños y niñas tienen derecho al desarrollo digno, esto implica cuidado, protección del curso normal y atención de calidad cuando hay alteraciones. La atención temprana planificada y ejecutada por un equipo profesional interdisciplinar y transdisciplinar cumple con estos objetivos dando respuesta oportuna a las necesidades de las alteraciones permanentes o transitorias del desarrollo (Peñafiel y col., 2003).

Existen diversos estudios sobre el impacto positivo que tienen los centros de cuidado diario en el desarrollo psicomotor de los niños y niñas de 0 a 5 años de edad, algunos criterios concluyen en que la temprana incorporación libra a los niños de peligros potenciales cuando el ambiente es peligroso sobre todo en zonas de pobreza, que provee de herramientas de aprendizaje para el afecto, la autonomía, la creatividad, la salud física (McWilliams y col., 2009). Cuando el currículo de los centros tiene como ejes el juego y el arte, los niños y niñas con alteraciones del desarrollo con o sin discapacidad pueden beneficiarse de la convivencia con los demás y de experiencias de intervención (Raina y col., 2005). Pero esto es posible cuando la calidad de atención es buena (Ackerman, 2008; Walsh y Gardner, 2005) en infraestructura, recursos humanos, programa educativo, participación familiar (Beneke, 2009) y tienen la licencia estatal de funcionamiento que cuida la calidad (Somers, 2011).

La calidad de la atención temprana se refiere al proceso de gestión para la protección del desarrollo normal o con alteraciones, para lograr calidad de vida de los niños, niñas y sus familias, esto es, satisfacción de las necesidades, potencialidad de las capacidades y alcance de las aspiraciones para lograr la actoría social con proyectos de vida individuales y colectivos (Observatorio de la Discapacidad, 2004; FEAPS, 1999; Michigan Department Education, 2006). Los centros de cuidado diario son espacios importantes para la implementación de la atención temprana y para que actúen con calidad deben seguir los elementos básicos de: (i) proceso de atención: evaluaciones periódicas del desarrollo, detección, derivación para el diagnóstico e intervención; (ii) capacidad profesional: personal que responda a las necesidades colectivas e individuales de los niños y niñas (Byington y Tannock, 2011; Oberhuermer, 2000); (iii) infraestructura: higiene, funcionalidad para el cuidado del desarrollo y la inclusión de los niños con necesidades especiales; (iv) participación familiar en el cuidado o en la intervención del desarrollo de los hijos e hijas (Douglas, 2011).

El estudio de cohorte en 30 niños de 3 centros de cuidado diario realizado en São Paulo, Brasil, en el 2005 teniendo como controles a niños y niñas que no asistieron a centros, que los que si lo hicieron demostraras mejores habilidades motoras gruesas y finas (Rezende y col., 2005). También existen estudios que indican que cuando la infraestructura no es adecuada y cuando los currículos se sustentan en un tipo de educación restrictiva, los niños y niñas tienen mayores problemas de conducta (Bradley y Vandel, 2007). El estudio en 41 estados de Estados Unidos en el 2005 reportó que todos los centros de cuidado infantil tenían currículos escolares educativos pero todos eran diferentes y algunos incompletos, no habían pautas para la inclusión de niños y niñas con necesidades educativas especiales (Scott y col., 2007), realidades similares han ocurrido en diferentes países sobre todo en los primeros años de implementación de los centros pero esto también ha llevado a la regulación de los mismos y acciones de mejoramiento, así la investigación experimental en 111 centros de cuidado diario en 5 condados de California (77 para la intervención y 38 para control) aplicando un programa de mejoramiento de las políticas y prácticas en salud por 3 años reportó como resultados el mejoramiento significativo de los centros intervenidos (Alkon y col., 2009); resultados que motivan a la planificación de estrategias de mejoramiento cuando se quiere lograr calidad.

El Ecuador implementó como meta para el período 2007-2010 aumentar la cobertura y la calidad de los proyectos de estimulación temprana y de educación inicial en los $\mathrm{CDI}^{1}$ públicos para niños y niñas de 0 a 5 años de edad. Meta que se propuso con el fin de proteger el derecho al desarrollo y para

\footnotetext{
${ }^{1}$ CDI: Centros de Desarrollo Infantil
} 
dar respuesta a la declaración del Observatorio de los derechos de la Niñez y Adolescencia en el Ecuador (2005) de que cerca de la mitad de los niños y niñas del quintil 1 y 2 de pobreza tienen serios retrasos en su desarrollo motriz, verbal y social (UNICEF, 2006). Reportes estatales mencionan que para el año 2009 el 49,12\% de los niños y niñas ecuatorianas no iban a ningún CDI y de los que asistían, el 50,88\% acudía a algún programa público del Ministerio de Educación o del INNFA-MIES; sin embargo, los informes indican que estos datos son imprecisos porque la evidencia no ha estado disponible ni organizada (Educiudadania, 2011). Para la consecución de la meta el estado ecuatoriano reglamentó que los CDI (CCNN, 2007; INNFA, 2007) cumplan con: (i) la obtención de la legalidad a través del Registro de Entidades de Atención en el Concejo Cantonal de la NNA garantizando los mejores estándares de atención (CCNNA, 2009); (ii) el seguimiento del Referente Curricular de la Educación Inicial con el fin de homogenizar la atención; (iii) cumplimiento de estándares de calidad; (iv) evaluación periódica del desarrollo psicomotor a través de la escala abreviada de Nelson-Ortiz (1999); y (v) la inclusión de niños y niñas con necesidades especiales.

La escala abreviada de Nelson-Ortiz (1999) evalúa el desarrollo psicomotor del niño en cuatro áreas: motriz grueso, motriz fino, desarrollo del lenguaje y audición, y personal social. El test, que fue estandarizado en niños colombianos, permite clasificar al sujeto de evaluación según su puntaje que se ubica por la edad en: alerta, medio, medio alto y alto. Para la utilización de esta guía el INNFA preparó al personal de los CDI públicos de manera que se pueda detectar tempranamente a los niños que requieran ser derivados (Rosero, 2009).

Sin embargo, a pesar de las políticas, las metas y los proyectos al realizar un acercamiento en el año 2009 a los CDI se pudo observar que el personal no era profesional, que en los documentos oficiales del Referente Curricular de la educación inicial, del Manual de Desarrollo del INNFA se exponen los parámetros para educar a los niñosy los protocolos de evaluación, pero no se aclaran las acciones a seguir cuando se detecta alerta en el desarrollo. Estas dificultades hacen que los niños y niñas con retrasos o alteraciones del desarrollo no sean detectados, no sean registrados, no consten en las estadísticas nacionales, y lo que es peor, no tengan la atención temprana que necesitan, aumentando en el futuro la deserción escolar por problemas de aprendizaje, el maltrato infantil y la exclusión en todos los espacios cotidianos. El informe del Ministerio de Coordinación de Desarrollo Social de noviembre del año 2009 indica que solo el 17\% de los niños de los CDI tienen un desarrollo adecuado para la edad (Rosero, 2009), es decir el 83\% tendría alerta en su desarrollo global, datos muy alarmantes. El estudio de prevalencia en el año 2009 en los CDI urbanos de Cuenca, realizado por un equipo de profesionales expertos en desarrollo, reporta que el $11 \%$ de los niños y niñas de 0 a 5 años de edad tuvieron retraso del desarrollo psicomotor y de ellos el 5\% discapacidad (Huiracocha y col., 2012). Los datos ecuatorianos expuestos hacen pensar que en los CDI la calidad de atención temprana no es la que se requiere y que esta impacta sobre la frecuencia de los retrasos del desarrollo. Por otro lado, si el personal no es profesional queda la duda sobre la calidad de la evaluación. La investigación en Connecticut en el 2009 que estudió las tasaciones hechas por los maestros de las habilidades de los niños preescolares, encontró que los maestros deben tener buena preparación y entrenamiento para darle el valor que corresponde al indicador, aun así las calificaciones dependen de lo que cada profesor quiera evaluar y qué teoría del desarrollo está implícito en cada uno de ellos, por esto menciona que la gran tarea de evaluar psicométricamente el desarrollo psicomotor en edades tempranas es complejo (Goldstein y McCoach, 2011).

El objetivo general de este estudio transversal fue determinar la relación entre desarrollo psicomotor de los niños y niñas de 0 a 5 años de edad y la calidad individual de atención temprana que reciben los niños en los Centros de Desarrollo Infantil urbanos de Cuenca. Los objetivos específicos fueron: (i) clasificar a los CDI según el tipo (públicos y privados), registro legal, integración a niños y niñas con discapacidad y calidad de atención temprana (muy buena, buena, regular y mala); (ii) clasificar el desarrollo de los niños con la escala abreviada de Ortiz (1999) en normal y alerta; y (iii) relacionar la alerta en el desarrollo psicomotor con la calidad individual en atención temprana de cada niño. 


\section{MATERIALES Y MÉTODOS}

\subsection{Población y entorno}

El Universo fue de 166 CDI regulares de los cuales el 25,9\% fueron públicos y el 74,1\% privados, con un total de 8145 niños y niñas de 0 a 5 años de edad de agosto a diciembre del 2009. La muestra clúster (elegida por causas económicas y logísticas) fue de 30 CDI que se seleccionaron aleatoriamente según el porcentaje presentado en el universo. Para la muestra de los niños se consideró el 5\% de retrasos del desarrollo, 3\% de inferencia, el 95\% de confianza y se calculó en el Stat Calc de EPI- INFO 3.5.1, consiguiendo 432 niños y niñas, al que se le añadió el $10 \%$ de pérdidas $(\mathrm{n}=475)$. Los niños y niñas de la muestra fueron tomados de los 30 CDI sorteados de manera proporcional a como se presentaron en el universo según el tipo de centro y el sexo. El sorteo se hizo con el software EpiData 3.1.

Los criterios de inclusión para los CDI fueron ser centros urbanos que atienden en jornadas entre 4 y 8 horas diarias a niños y niñas de 0 a 5 años de edad preferentemente sin discapacidad y que en el período de la investigación no clausuraran. El criterio de exclusión fue atender solo a niños con discapacidad. Los criterios de inclusión para los niños fueron tener entre 0 y 5 años de edad, asistencia regular y que el representante firme el consentimiento informado. El criterio de exclusión fue tener el diagnóstico previo de discapacidad, participar en otra investigación y la actitud persistente de no colaboración del niño o niña. Cuando un niño faltó o estuvo enfermo se regresó al otro día para hacer la evaluación y cuando no fue posible se consideró como perdido. Gracias a la colaboración de los responsables y maestros, y las actuaciones del equipo técnico de investigación (actividades de ambientación, comunicación y buen trato con los niños, padres y maestras que impartió confianza) el número total de niños y niñas que ingresaron a la investigación fue de 447 y 28 casos perdidos.

\subsection{Variables}

Las variables de estudio fueron las siguientes:

$\checkmark$ Tipo de CDI: institución de cuidado diario o de educación inicial para niños y niñas de 4 a 8 horas diarias. Es público, si el financiamiento procede del estado, o es privado.

$\checkmark$ Registro Legal: documento que acredite el registro en el Concejo Cantonal de la NNA luego del cumplimiento de requisitos y de estándares de calidad. Es si cumple o no cumple.

$\checkmark$ Integración: si el CDI tiene matriculado a un niño o niña con discapacidad que asiste regularmente.

$\checkmark$ Grupo etario del niño o niña: lactantes hasta los 2 años y preescolares de 3 a 5 años.

$\checkmark$ Sexo, diferencia de genitales: Es: masculino o femenino.

$\checkmark$ Desarrollo psicomotriz: logros en cada etapa medidos por la escala abreviada de Ortiz (1999) con puntaje total que se ubica en una tabla de percentiles que clasifica el desarrollo en: alerta (bajo el percentil 10), medio (entre los percentiles 10 y 50), medio alto (entre los percentiles 50 y 90) y alto (más del percentil 90).

$\checkmark$ Alerta por áreas del desarrollo: puntuación que en alguna de las áreas se ubica en el cuadro de calificación como alerta. Es: alerta motriz gruesa, alerta motriz fina, alerta lenguaje y audición, y alerta en personal social.

$\checkmark$ Alerta del desarrollo psicomotor: puntaje en una, en varias o en el puntaje total que se ubica en la tabla de calificación como alerta. Es: alerta o desarrollo normal.

$\checkmark$ Calidad individual de atención temprana: cuidado del desarrollo a través de un proceso integral que contempla los siguiente indicadores de calidad: proceso de atención, preparación profesional, infraestructura y participación familiar. Es: muy buena (cumple con los 4 indicadores), buena (cumple con 3), regular (cumple con 2) y mala (cumple con 1 o con ninguno).

$\checkmark$ Cumplimiento individual de la atención temprana es cumple (cuando la calidad de atención temprana es muy buena o buena), no cumple (cuando la calidad de atención temprana es regular o mala).

$\checkmark$ Calidad individual del proceso de atención, se considera los siguientes indicadores: (i) constancia del registro, en el expediente del niño, de la evaluación realizada en el CDI; (ii) constancia del 
registro del niño o niña que calificó en la evaluación del CDI como alerta de todos los siguientes: detección, derivación y adaptaciones curriculares. Es: cumple con la calidad (presencia de todos los indicadores de acuerdo al tipo de desarrollo) no cumple con la calidad (ausencia de alguno de los indicadores).

$\checkmark$ Calidad individual de la preparación profesional: el título profesional de tercer nivel en el área de trabajo de la maestra o del maestro del niño de la muestra evaluado por el equipo de la investigación. Es: cumple con la calidad o no cumple con la calidad.

$\checkmark$ Calidad individual de la infraestructura: cuando el niño evaluado se encuentra en un aula que cumple con los estándares de calidad de infraestructura por los criterios de espacios funcionales, seguridad ambiental, higiene y ausencia de barreras arquitectónicas en el acceso. Es: cumple con la calidad (con todos los indicadores) o no cumple con la calidad (falta alguno de los indicadores).

$\checkmark$ Calidad individual de la participación familiar: cuando el padre del niño evaluado cumplió con los indicadores de participación en las tareas obligatorias-voluntarias del centro y con apoyar en las tareas de su hijo e hija. Es: cumple (con los dos indicadores) o no cumple (falta un indicador).

$\checkmark$ Calidad de atención temprana del CDI es: muy buena (cuando del $80 \%$ o más de los niños cumplen con la calidad de atención temprana), buena (cuando del 60 al 79\% de los niños cumplen la calidad de atención temprana), regular (cuando del 40 al 59\% de los niños cumplen la calidad de atención temprana), mala (cuando del 39\% o menos de los niños cumplen la calidad de atención temprana).

$\checkmark$ Calidad del CDI en el proceso de atención es: muy buena (cuando del $80 \%$ o más de los niños cumplen con la calidad del proceso de atención), buena (cuando del 60 al 79\% de los niños cumplen la calidad del proceso de atención), regular (cuando del 40 al 59\% de los niños cumplen la calidad del proceso de atención), mala (cuando del 39\% o menos de los niños cumplen la calidad del proceso de atención).

$\checkmark$ Calidad del CDI en la preparación profesional es: muy buena (cuando del $80 \%$ o más de los niños cumplen con la calidad de la preparación profesional), buena (cuando del 60 al $79 \%$ de los niños cumplen la calidad de la preparación profesional), regular (cuando del 40 al 59\% de los niños cumplen la calidad de la preparación profesional), mala (cuando del $39 \%$ o menos de los niños cumplen la calidad de la preparación profesional).

$\checkmark$ Calidad del CDI en la infraestructura es: muy buena (cuando del $80 \%$ o más de los niños cumplen con la calidad de la infraestructura), buena (cuando del 60 al $79 \%$ de los niños cumplen la calidad de la infraestructura), regular (cuando del 40 al 59\% de los niños cumplen la calidad de infraestructura), mala (cuando del 39\% o menos de los niños cumplen la calidad de la infraestructura).

$\checkmark$ Calidad del CDI en la participación familiar es: muy buena (cuando del $80 \%$ o más de los niños cumplen con la calidad en la participación familiar), buena (cuando del 60 al $79 \%$ de los niños cumplen la calidad en la participación familiar), regular (cuando del 40 al 59\% de los niños cumplen la calidad en la participación familiar), mala (cuando del 39\% o menos de los niños cumplen la calidad en la participación familiar).

Se consideró como variable dependiente la alerta del desarrollo psicomotor y como variables independientes la calidad individual de atención temprana, la calidad individual del proceso de atención, la calidad individual de la preparación profesional, la calidad individual de la infraestructura, y la calidad individual de la participación familiar.

\subsection{Instrumentos y procedimientos de recolección de la información}

Se aplicó un formulario al que se le añadió los anexos instructivos de la evaluación. Previo al inicio de la investigación se realizaron talleres al personal de los CDI, se preparó y se estandarizó a los 7 equipos de evaluación que estuvieron conformados por 1 coordinadora y 2 estimuladoras. Se encargó a una profesional para la supervisión diaria de la calidad de recolección de los datos, la directora garantizó la comunicación asertiva y la logística durante el proceso. Se realizó el pilotaje en 4 CDI y en 30 niños sorteados y que no fueron parte de la muestra. La recolección tomó 4 meses. El tiempo aproximado de cada niño o niña fue de una hora siguiendo los pasos de: (i) llenado con la maestra de los datos de edad, sexo del niño o niña; (ii) revisión del expediente del niño para constatar el registro 
de la evaluación, de la detección, derivación y adaptación curricular, la participación de los padres en el apoyo escolar del niño; (iii) revisión del título académico del profesor o de la profesora del niño o niña evaluado; (iv) observación del aula del niño para calificar la infraestructura; (v) evaluación del desarrollo psicomotor del niño; (vi) calificación de la calidad del CDI. Como parte del control de calidad se aplicó a 23 niños (5\%), sorteados aleatoriamente de la muestra, una nueva evaluación dentro de los siete días siguientes a la primera. Con los resultados de la evaluación se escribieron informes para cada uno de los niños y niñas y se entregaron a los maestros y padres de familia, derivando a aquellos con alerta en el desarrollo para el diagnóstico y plan de intervención.

\subsection{Análisis estadístico}

No existieron valores perdidos. En el análisis descriptivo con las variables cuantitativas continuas (edad en meses y puntaje de desarrollo) se comprobó que no presentaron un patrón normal y se seleccionó como medidas de tendencia central y de distribución a la mediana, el mínimo, el máximo y los percentiles; con las variables cualitativas se emplearon frecuencias y porcentajes. En las tablas descriptivas para saber si hay diferencia significativa en las variables continuas se empleó la prueba de U de Mann Whitney y en las cualitativas la diferencia de proporciones. Se obtuvo la prevalencia de los niños y niñas con alerta en el desarrollo con el intervalo de confianza al 95\% con una prueba de proporción. Para la relación de la variable dependiente con las independientes dicotómicas el Chi sq, razón de prevalencia (RP) con intervalos de confianza al 95\%; se aplicó corrección de Yates cuando al menos el valor de una frecuencia esperada fue menos que 5, y también se utilizó Mantel Haenszel para diferenciar o sumar los estratos.

\section{RESULTADOS Y DISCUSIÓN}

Los resultados de esta investigación son un buen referente para evaluar los alcances obtenidos para cumplir con la meta del Estado Ecuatoriano para el período 2007-2010, el de aumentar la cobertura y la calidad del cuidado y de la atención temprana del desarrollo en los CDI. De los 166 CDI del universo el 26,7\% fueron públicos y el 73,3\% privados. De los 30 centros de la muestra el 23,3\% tuvo el registro legal, el 10\% integró a niños y niñas con discapacidad, el 23,3\% presentó una calidad entre muy buena $(20 \%)$ y buena $(3,3 \%)$ y el $76,7 \%$ tienen mala calidad de atención temprana (Tabla 1$)$, esto significa que solo 6 de los 30 centros calificó en todas las dimensiones como muy buena, que un centro tuvo tres y que 23 cumplió con una o con ninguna de las dimensiones de proceso de atención, preparación profesional, infraestructura y participación familiar.

Estos resultados contradicen a los presentados en el informe de Educiudadanía que evalúa los avances del Plan Decenal de Educación donde para el año 2009 la cobertura de los niños atendidos por las instituciones públicas fue del 50,88\% y para el año 2010 del 50,17\%. Esta diferencia se debe a la metodología de la recolección de los datos, puesto que mientras el mismo informe declara que los datos no son precisos, esta investigación realizó un mapeo minucioso de los CDI urbanos de Cuenca e hizo una base de datos de los niños y niñas (Educiudadanía, 2011). El no registro legal puede deberse a que a finales del 2008 recién se organizaron nacionalmente en los Concejos Cantonales de la Niñez y Adolescencia los pasos para el registro de las entidades, el hecho es que en el momento de la recolección de la información, la mayoría de las instituciones no conocían de este requisito (CNNA, 2009).

Al tomar las dimensiones de la calidad de manera individual se observa que no hay diferencia significativa al comparar la calidad entre los CDI públicos y privados (Tabla 2); aproximadamente el $50 \%$ de los CDI tienen calidad muy buena en proceso de atención, preparación profesional, infraestructura, participación familiar pero el 23,3\% de los CDI no cumplen con proceso de atención, así como el 50\% de los CDI no tienen personal profesional, el 50\% los CDI no cumplen con espacios funcionales, saneamiento, higiene y tienen barreras arquitectónicas (infraestructura), en el 43,3\% los padres de los niños y niñas no cumplen con las actividades obligatorias-voluntarias organizadas por 
Tabla 1. Características de los 30 Centros de Desarrollo Infantil (CDI) Urbanos de Cuenca, objeto de la investigación (2009).

\begin{tabular}{lrr}
\hline Características & \multicolumn{2}{c}{ Total } \\
\hline Tipo de centro & & \\
Público & 8 & 26,7 \\
Privado & 22 & 73,3 \\
Total & 30 & 100,0 \\
Registro Legal & & \\
Si & 7 & 23,3 \\
No & 23 & 76,7 \\
Total & 30 & 100,0 \\
Integración a niños y niñas con discapacidad & & \\
Si & 3 & 10,0 \\
No & 37 & 90,0 \\
Total & 27 \\
Calidad de la Atención Temprana & 30 & 100,0 \\
Muy buena & & \\
Buena & \multicolumn{2}{c}{} \\
Regular & 6 & 20,0 \\
Mala & 1 & 3,3 \\
Total & 0 & 0,0 \\
\hline
\end{tabular}

los centros ni ayudan en la tarea de sus hijos e hijas (participación familiar). La mala calidad de atención puede deberse a que desde el año 1999 al 2008 existieron una variedad de modalidades públicas de atención a niños de 0 a 5 años de edad con diversidad de enfoques y variedad de estrategias por lo que desde el año 2008 hasta la actualidad los CDI se están organizando bajo dos modalidades: estimulación temprana para menores de 3 años y educación inicial para niños y niñas de 3 a 4 años de edad; sin embargo, todavía los centros no están claros en qué referente curricular utilizar y la escala abreviada de Ortiz (1999) ha sido retirada (luego de varios años de entrenamiento de las cuidadoras de los CDI), se continúa sin un protocolo de derivación y atención a los niños y niñas con retraso del desarrollo con o sin discapacidad (Educiudadania, 2011) lo que se refleja en el bajo porcentaje de integración a pesar que la ley manda que por cada aula debe haber 2 niños o niñas con necesidades especiales. Desde el año 2010 existe el programa del Ministerio de Educación denominado Proyecto de Educación Inicial de Calidad y Calidez y el Proyecto de desarrollo profesional educativo SiProfe pero todavía no se conocen los resultados de estos programas (Ministerio de Educación, 2012). Todas estas realidades se transparentan en la calidad de la atención temprana que los niños y niñas reciben en los CDI.

De los 447 el 30\% (134) niños procedieron de los CDI públicos y el 70\% (313) de los privados, el $53 \%$ (237) fueron niños y el $47 \%$ (210) niñas, con una mediana de 49,9 y 49 respectivamente (p > 0,05); 27 fueron lactantes (6\%) y 420 (94\%) preescolares (Tabla 3). La mediana de puntaje de desarrollo evaluado por la escala abreviada de Ortiz (1999) fue de 111 para los niños y de 113 para las niñas $(p>0,05)$. Del total de niños y niñas el $26 \%$ (116) no fueron evaluados en sus centros. El 10,5\% (IC 95\%: 7,8 a 13,7\%) presentó alerta en el desarrollo psicomotor ya sea porque tuvo alerta en una, en varias o en todas las áreas, $24(3,6 \%)$ de los 47 niños presentaron alerta en el área motora gruesa, 16 $(3,6 \%)$ en la motora fina, $29(6,5 \%)$ en lenguaje y audición, $29(6,5 \%)$ en personal social y $21(4,7 \%)$ en todas las áreas. No existió diferencia significativa de las proporciones entre los CDI públicos y 
Tabla 2. Características de calidad de los 30 CDI Urbanos de Cuenca (2009).

\begin{tabular}{llllllll}
\hline \multirow{2}{*}{ Características de calidad } & \multicolumn{2}{c}{ Públicos } & \multicolumn{2}{c}{ Privados } & \multicolumn{2}{c}{ Total } & \multirow{2}{*}{$\mathrm{p}$} \\
\cline { 2 - 6 } & No. & $\%$ & No. & $\%$ & No. & $\%$ & \\
\hline
\end{tabular}

Calidad de proceso de atención

$\begin{array}{lrrrrrrr}\text { Muy buena } & 4 & 50,0 & 13 & 59,1 & 17 & 56,7 & 0,658 \\ \text { Buena } & 2 & 25,0 & 1 & 4,5 & 3 & 10,0 & 0,166 \\ \text { Regular } & 0 & 0,0 & 3 & 13,7 & 3 & 10,0 & 0,545 \\ \text { Mala } & 2 & 25,0 & 5 & 22,7 & 7 & 23,3 & 0,898 \\ \text { Total } & 8 & 100,0 & 22 & 100,0 & 30 & 100,0 & \end{array}$

Calidad de preparación profesional

$\begin{array}{lrrrrrrr}\text { Muy buena } & 4 & 50,0 & 11 & 50,0 & 15 & 50,0 & 1,00 \\ \text { Buena } & 0 & 0,0 & 0 & 0,0 & 0 & 0,0 & \\ \text { Regular } & 0 & 0,0 & 0 & 0,0 & 0 & 0,0 & \\ \text { Mala } & 4 & 50,0 & 11 & 50,0 & 15 & 50,0 & 0,370 \\ \text { Total } & 8 & 100,0 & 22 & 100,0 & 30 & 100,0 & \end{array}$

Calidad de la infraestructura

$\begin{array}{lrrrrrrr}\text { Muy buena } & 3 & 50,0 & 11 & 50,0 & 14 & 46,7 & 0,535 \\ \text { Buena } & 0 & 0,0 & 1 & 4,5 & 1 & 3,3 & 0,306 \\ \text { Regular } & 0 & 0,0 & 0 & 0,0 & 0 & 0,0 & \\ \text { Mala } & 5 & 50,0 & 10 & 45,5 & 15 & 50,0 & 0,397 \\ \text { Total } & 8 & 100,0 & 22 & 100,0 & 30 & 100,0 & \end{array}$

Calidad de la participación familiar

\begin{tabular}{lrrrrrrr} 
Muy buena & 5 & 62,5 & 12 & 54,6 & 17 & 56,7 & 0,693 \\
Buena & 0 & 0,0 & 0 & 0,0 & 0 & 0,0 & \\
Regular & 0 & 0,0 & 0 & 0,0 & 0 & 0,0 & \\
Mala & 3 & 37,5 & 10 & 45,5 & 13 & 43,3 & 0,693 \\
Total & 8 & 100,0 & 22 & 100,0 & 30 & 100,0 & \\
\hline
\end{tabular}

Tabla 3. Características de los 447 niños y niñas en los 30 Centros de Desarrollo Infantil Urbanos de Cuenca (2009).

\begin{tabular}{lrrrrrr}
\hline \multirow{2}{*}{ Variables } & \multicolumn{6}{c}{ Masculino } \\
\cline { 2 - 7 } Edad & Mínimo & Mediana & Máximo & PC 10 & PC 50 & PC 90 \\
\hline $\begin{array}{l}\text { Puntaje de } \\
\text { desarrollo según } \\
\begin{array}{l}\text { Nelson-Ortiz } \\
\hline\end{array}\end{array}$ & 26,8 & 111,0 & 120,0 & 74,6 & 111,0 & 118,0 \\
\hline
\end{tabular}

\begin{tabular}{|c|c|c|c|c|c|c|c|}
\hline \multirow{2}{*}{ Variables } & \multicolumn{6}{|c|}{ Femenino } & \multirow{2}{*}{$\mathrm{p}$} \\
\hline & Mínimo & Mediana & Máximo & PC 10 & PC 50 & PC 90 & \\
\hline Edad & 14,0 & 49,0 & 60,0 & 29,3 & 49,2 & 58,3 & 0,845 \\
\hline $\begin{array}{l}\text { Puntaje de } \\
\text { desarrollo según } \\
\text { Nelson-Ortiz }\end{array}$ & 45,0 & 113,0 & 128,0 & 80,0 & 113,0 & 118,0 & 0,108 \\
\hline
\end{tabular}


privados. La prevalencia de niños y niñas con alerta en el desarrollo psicomotor determinada por la evaluación de equipos especializados de esta investigación (Tabla 4) está más acorde con la literatura internacional (Avaria, 2005) que con los datos nacionales (UNICEF, 2006; Rosero, 2009) que estuvieron entre el $50 \%$ y el $83 \%$. Esta investigación indica que probablemente los procesos de evaluación no fueron los más adecuados, ratificándose lo que el estudio de Connecticut del 2009 recomendó, el de preparar y entrenar a profesionales para la complicada tarea de evaluar el desarrollo psicomotor de los niños y niñas (Goldstein, 2011).

Tabla 4. Desarrollo psicomotor de los 447 niños y niñas en los 30 Centros de Desarrollo Infantil Urbanos de Cuenca (2009).

\begin{tabular}{|c|c|c|c|c|c|c|c|}
\hline \multirow{2}{*}{ Desarrollo psicomotor } & \multicolumn{2}{|c|}{ Público } & \multicolumn{2}{|c|}{ Privado } & \multicolumn{2}{|c|}{ Total } & \multirow{2}{*}{$\mathrm{p}$} \\
\hline & No & $\%$ & No & $\%$ & No. & $\%$ & \\
\hline \multicolumn{8}{|l|}{ Desarrollo psicomotor } \\
\hline $\begin{array}{l}\text { Alerta en una, en varias o en } \\
\text { todas las áreas }\end{array}$ & 20 & 14,9 & 27 & 8,6 & 47 & 10,5 & 0,063 \\
\hline Normal & 114 & 85,1 & 286 & 91,4 & 400 & 89,5 & \\
\hline Total & 134 & 100,0 & 313 & 100,0 & 447 & 100,0 & \\
\hline \multicolumn{8}{|l|}{ Desarrollo motor grueso } \\
\hline Alerta & 7 & 5,2 & 9 & 2,9 & 24 & 3,6 & 0,266 \\
\hline Normal & 127 & 94,8 & 304 & 97,1 & 423 & 94,6 & \\
\hline Total & 134 & 100,0 & 313 & 100,0 & 447 & 100,0 & \\
\hline \multicolumn{8}{|l|}{ Desarrollo motor fino } \\
\hline Alerta & 9 & 6,7 & 15 & 4,8 & 16 & 3,6 & 0,492 \\
\hline Normal & 125 & 93,3 & 298 & 95,2 & 431 & 96,4 & \\
\hline Total & 134 & 100,0 & 313 & 100,0 & 447 & 100,0 & \\
\hline \multicolumn{8}{|l|}{ Desarrollo lenguaje y audición } \\
\hline Alerta & 12 & 9,0 & 17 & 5,4 & 29 & 6,5 & 0,207 \\
\hline Normal & 122 & 91,0 & 296 & 94,6 & 418 & 93,5 & \\
\hline Total & 134 & 100,0 & 313 & 100,0 & 447 & 100,0 & \\
\hline \multicolumn{8}{|l|}{ Desarrollo personal social } \\
\hline Alerta & 10 & 7,5 & 19 & 6,1 & 29 & 6,5 & 0,75 \\
\hline Normal & 124 & 92,5 & 294 & 93,9 & 418 & 93,5 & \\
\hline Total & 134 & 100,0 & 313 & 100,0 & 447 & 100,0 & \\
\hline \multicolumn{8}{|l|}{$\begin{array}{l}\text { Alerta en todas las áreas del } \\
\text { desarrollo }\end{array}$} \\
\hline $\mathrm{Si}$ & 8 & 6,0 & 13 & 4,2 & 21 & 4,7 & 0,465 \\
\hline No & 126 & 94,0 & 300 & 95,8 & 426 & 95,3 & \\
\hline Total & 134 & 100,0 & 313 & 100,0 & 447 & 100,0 & \\
\hline
\end{tabular}

De los 49 niños y niñas con alerta el 79,6\% (39) no tuvo el registro de detección en su historial, el 93,9\% (46) no fue derivado para diagnóstico y plan de intervención, el 93,9\% (46) no tuvo una adaptación curricular por su necesidad especial, esto no fue diferente ni en los públicos ni en los privados (Tabla 5). Los resultados se ratifican cuando los datos demuestran que el no cumplimiento de la calidad del proceso de atención temprana tiene relación con alerta en el desarrollo psicomotor de los 
Tabla 5. Cumplimiento de la calidad individual de la atención de los 447 niños y niñas en los 30 Centros de Desarrollo Infantil Urbanos de Cuenca (2009).

\begin{tabular}{|c|c|c|c|c|c|c|c|}
\hline \multirow{2}{*}{ Calidad } & \multicolumn{2}{|c|}{ Público } & \multicolumn{2}{|c|}{ Privado } & \multicolumn{2}{|c|}{ Total } & \multirow{2}{*}{$\mathrm{p}$} \\
\hline & No & $\%$ & No & $\%$ & No. & $\%$ & \\
\hline \multicolumn{8}{|c|}{ Evaluación del desarrollo } \\
\hline No cumple & 28 & 20,9 & 88 & 28,1 & 116 & 26,0 & 0,126 \\
\hline Cumple & 106 & 79,1 & 225 & 71,9 & 331 & 74,0 & \\
\hline Total & 134 & 100,0 & 313 & 100,0 & 447 & 100,0 & \\
\hline \multicolumn{8}{|l|}{ Detección } \\
\hline No cumple & 18 & 90,0 & 21 & 72,4 & 39 & 79,6 & 0,167 \\
\hline Cumple & 2 & 10,0 & 8 & 27,6 & 10 & 20,4 & \\
\hline Total & 20 & 100,0 & 29 & 100,0 & 49 & 100,0 & \\
\hline \multicolumn{8}{|l|}{ Derivación } \\
\hline No cumple & 20 & 100,0 & 26 & 89,7 & 46 & 93,9 & 0,260 \\
\hline Cumple & 0 & 0,0 & 3 & 10,3 & 3 & 6,1 & \\
\hline Total & 20 & 100,0 & 29 & 100,0 & 49 & 100,0 & \\
\hline \multicolumn{8}{|c|}{ Adaptaciones curriculares } \\
\hline No cumple & 20 & 100,0 & 26 & 89,7 & 46 & 93,9 & 0,260 \\
\hline Cumple & 0 & 0,0 & 3 & 10,3 & 3 & 6,1 & \\
\hline Total & 20 & 100,0 & 29 & 100,0 & 49 & 100,0 & \\
\hline \multicolumn{8}{|c|}{ Espacios funcionales } \\
\hline No cumple & 61 & 45,5 & 25 & 8,0 & 86 & 19,2 & 0,00 \\
\hline Cumple & 73 & 54,5 & 288 & 92,0 & 361 & 80,8 & \\
\hline Total & 134 & 100,0 & 313 & 100,0 & 447 & 100,0 & \\
\hline \multicolumn{8}{|c|}{ Barreras arquitectónicas } \\
\hline Tiene & 28 & 20,9 & 94 & 30,0 & 122 & 27,3 & 0,049 \\
\hline No tiene & 106 & 79,1 & 219 & 70,0 & 325 & 72,7 & \\
\hline Total & 134 & 100,0 & 313 & 100,0 & 447 & 100,0 & \\
\hline \multicolumn{8}{|c|}{ Seguridad ambiental } \\
\hline No cumple & 70 & 52,2 & 54 & 17,3 & 124 & 27,7 & 0,000 \\
\hline Cumple & 64 & 47,8 & 259 & 82,7 & 323 & 72,3 & \\
\hline Total & 134 & 100,0 & 313 & 100,0 & 447 & 100,0 & \\
\hline \multicolumn{8}{|l|}{ Higiene } \\
\hline No cumple & 25 & 18,7 & 0 & 0,0 & 25 & 5,6 & 0,000 \\
\hline Cumple & 109 & 81,3 & 313 & 100,0 & 422 & 94,4 & \\
\hline Total & 134 & 100,0 & 313 & 100,0 & 447 & 100,0 & \\
\hline \multicolumn{8}{|c|}{$\begin{array}{l}\text { Apoyo en las actividades del } \\
\text { centro }\end{array}$} \\
\hline No cumple & 56 & 41,8 & 111 & 35,5 & 167 & 37,4 & 0,240 \\
\hline Cumple & 78 & 58,2 & 202 & 64,5 & 280 & 62,6 & \\
\hline Total & 134 & 100,0 & 313 & 100,0 & 447 & 100,0 & \\
\hline \multicolumn{8}{|c|}{ Apoyo en las tareas con los hijos e hijas } \\
\hline No cumple & 19 & 14,2 & 0 & 0,0 & 19 & 4,3 & 0,000 \\
\hline Cumple & 115 & 85,8 & 313 & 100,0 & 428 & 95,7 & \\
\hline Total & 134 & 100,0 & 313 & 100,0 & 447 & 100,0 & \\
\hline
\end{tabular}


niños y niñas ( $\mathrm{p}<0,05$ ) (Tabla 6). El 19,2\% (86) de los niños no disfrutó de espacios funcionales, el 27,3\% (122) sufrió por tener barreras físicas para la realización de sus actividades lúdicas, el 27,7\% (124) no gozó de seguridad ambiental, el 5,6\% (25) no tuvo un espacio limpio; los niños y niñas con problemas en su desarrollo estuvieron mayormente en un espacio con una infrastructura inadecuada. En el 72,7\% (325) de los niños, sus maestras o maestros no tuvieron título profesional en el área del trabajo (desarrollo infantil) pero cuando se relaciona con alerta en el desarrollo no se encuentra relación significativa $(\mathrm{p}>0,05)$ (Tabla 6) lo que dirige a pensar que más impacto sobre el desarrollo del niño puede tener la modalidad, las estrategias y las planificaciones curriculares educativas. En el $37,4 \%$ (167) de los niños sus padres no apoyaron en las actividades obligatorias y voluntarias programadas por los centros, en el 4,3\% de los niños sus padres no apoyaron en las tareas o en las responsabilidades de sus hijos e hijas, siendo mayor el problema en los privados (Tabla 5). En la relación no cumplimiento de la calidad de participación familiar con alerta en el desarrollo existe significancia $(\mathrm{p}<0,05)$ (Tabla 6), es decir existieron más niños con dificultades en el desarrollo en los hogares cuyos padres no les apoyan.

Tabla 6. Relación de alerta en el desarrollo psicomotor y calidad de atención temprana.

\begin{tabular}{|c|c|c|c|c|c|c|c|c|c|}
\hline \multirow{2}{*}{$\begin{array}{c}\text { Calidad de atención } \\
\text { temprana }\end{array}$} & \multicolumn{2}{|c|}{$\begin{array}{l}\text { Alerta en el } \\
\text { desarrollo }\end{array}$} & \multicolumn{2}{|c|}{$\begin{array}{c}\text { Desarrollo } \\
\text { normal }\end{array}$} & \multicolumn{2}{|c|}{ Total } & \multirow[t]{2}{*}{$\mathrm{p}$} & \multirow[t]{2}{*}{$\mathrm{RP}$} & \multirow[t]{2}{*}{ IC $95 \%$} \\
\hline & No. & $\%$ & No. & $\%$ & No. & $\%$ & & & \\
\hline Proceso de atención & & & & & & & 0,000 & 33,42 & 9,7 a 137,6 \\
\hline No cumple con & 44 & 93, & 122 & 30 , & 166 & 37,1 & & & \\
\hline Cumple con calidad & 3 & 6,4 & 278 & 69 , & 281 & 61,9 & & & \\
\hline Total & 47 & 100 & 400 & 100 & 447 & 100,0 & & & \\
\hline Preparación profesional & & & & & & & $\begin{array}{l}0,073 \\
0,00_{\mathrm{M}}\end{array}$ & $\begin{array}{r}0,60 \\
2,1_{\mathrm{MH}}\end{array}$ & $\begin{array}{r}0,3 \text { a } 1,1 \\
2,6 \text { a } 2,5_{\mathrm{MH}}\end{array}$ \\
\hline No cumple con & 29 & 61, & 296 & 74 , & 325 & 72,7 & & & \\
\hline Cumple con calidad & 18 & 38, & 104 & 26 , & 122 & 27,3 & & & \\
\hline Total & 47 & 100 & 400 & 100 & 447 & 100,0 & & & \\
\hline Infraestructura & & & & & & & $\begin{array}{l}0,017 \\
0,00_{M}\end{array}$ & $\begin{array}{l}2,06 \\
2_{\mathrm{MH}}\end{array}$ & $\begin{array}{r}1,1 \text { a } 3,9 \\
2,6 \text { a } 5,7_{\mathrm{MH}}\end{array}$ \\
\hline No cumple con & 35 & 74 & 212 & 53 , & 247 & 55,3 & & & \\
\hline Cumple con calidad & 12 & 25 & 188 & 47 , & 200 & 44,7 & & & \\
\hline Total & 47 & 100 & 400 & 100 & 447 & 100,0 & & & \\
\hline Participación familiar & & & & & & & $\begin{array}{l}0,017 \\
0,00_{\mathrm{M}}\end{array}$ & $\begin{array}{r}2,06 \\
1,98_{\mathrm{M}}\end{array}$ & $\begin{array}{l}1,1 \text { a } 3,96 \\
2,5 \text { a } 4_{\mathrm{MH}}\end{array}$ \\
\hline No cumple con & 25 & 53, & 142 & 35 , & 167 & 37,4 & & & \\
\hline Cumple con calidad & 22 & 46, & 258 & 64 , & 280 & 62,6 & & & \\
\hline Total & 47 & 100 & 400 & 100 & 447 & 100,0 & & & \\
\hline Calidad de la atención $t$ & empra & & & & & & $\begin{array}{l}0,007 \\
0,00_{\mathrm{M}}\end{array}$ & $\begin{array}{r}4,89 \\
2,1_{\mathrm{MH}} \\
\end{array}$ & $\begin{array}{l}1,42 \text { a } 20,1 \\
2,6 \text { a } 4,2 \mathrm{MH}\end{array}$ \\
\hline No cumple con & 44 & 93, & 300 & 75 , & 344 & 77,0 & & & \\
\hline Cumple con calidad & 3 & 6,4 & 100 & 25 , & 103 & 23,0 & & & \\
\hline Total & 47 & 100 & 400 & 100 & 447 & 100,0 & & & \\
\hline
\end{tabular}

Por todos los datos expuestos se ratifica que existe relación entre la calidad de atención temprana y la alerta en el desarrollo $(\mathrm{p}<0,05)$ (Tabla 6), es decir existen más niños y niñas con alerta en el desarrollo en los centros donde la calidad de atención no se cumple o es mala, resultados que están de acuerdo con los estudios internacionales que subrayan que los centros de cuidado diario pueden potenciar el desarrollo cuando cumple los estándares de calidad (McWilliams y col., 2009; Raina y col., 2005) pero pueden causar efectos adversos cuando las circunstancias no son apropiadas, hechos que resultan más impactantes en los niños y niñas con alerta en el desarrollo que se quedan sin 
diagnóstico, sin plan de intervención y por lo tanto tienen un futuro incierto en las posibilidades de incorporarse a esta sociedad llena de retos y desafíos (Ackerman, 2008; Walsh y Gardner, 2005; Somers, 2011).

Una de las limitaciones del estudio fue no adentrarse en cómo se planifica, cómo son las condiciones de trato, cuáles son los significados culturales de los maestros en el cuidado del desarrollo y no indagar sobre factores familiares, biológicos, criterios que pueden tener mayor impacto en el desarrollo psicomotor de los niños y niñas. Otra de las limitaciones fue que al ser un estudio transversal no se puede definir relaciones causales por lo que se recomienda estudios analíticos.

\section{CONCLUSIONES}

La investigación llegó a determinar que la calidad de atención temprana que los niños y niñas de 0 a 5 años de edad reciben en los Centros de Desarrollo Infantil Urbanos de Cuenca está relacionada con la alerta del desarrollo psicomotor. El no cumplimiento de la evaluación del desarrollo en todos los niños, la no detección, la no derivación y la no realización de adaptaciones curriculares en aquellos con dificultades hace que el proceso de atención en la mayoría de los centros no sea adecuada; aunque el mayor porcentaje de cuidadoras de los centros no son profesionales, la preparación no tiene relación con alerta en el desarrollo psicomotor, no así la infraestructura y la participación familiar que al no ser cumplida sí se relaciona con los casos alerta. La prevalencia de los niños y niñas con alerta en el desarrollo fue similar a los datos reportados por la literatura internacional pero diferente a los nacionales. En el año 2009 los objetivos planteados por el estado para cumplir con la meta de aumentar la cobertura y la calidad de los centros parecen no haberse cumplido, se recomienda mejorar todas las regulaciones, las planificaciones que lleven a la optimización del proceso de atención, infraestructura, preparación profesional y participación familiar.

\section{AGRADECIMIENTOS}

Los autores agradecen a la Dirección de Investigación de la Universidad de Cuenca (DIUC), al Concejo Cantonal de la Niñez y Adolescencia, al Ministerio de Salud Pública, al Ministerio de Educación, al Ministerio de Bienestar Económico y Social, al Instituto de la Niñez y la Familia, a los Centros de Desarrollo Infantil, a las estudiantes de Tecnología Médica, a los padres de familia, a los niños y niñas; porque la concertación, el trabajo organizado permitió la realización de la investigación.

\section{BIBLIOGRAFIA}

Ackerman, D., 2008. La consultoría y un proyecto preliminar de tasación de calidad. ECRP Early Child Research Practice, 10(2). Descargado de http://ecrp.uiuc.edu/v10n2/ackerman-sp.html el 7 de abril 2009.

Alkon, A., J. Bernzweing, K. To, M. Wolf, J.F. Mackie, 2009 . CHild care health consultation improves health and safety polices and practices. Acad. Pediatr., 9(5), 366-70. Descargado de http://pesquisa.bvsalud.org/regional/resources/mdl-19640823\# en agosto 2010.

Avaria, M., 2005. Pediatría del desarrollo y comportamiento. Rev. Ped. Elec [en linea], 2(1). Descargado de http://www.revistapediatria.cl/vol2numl/14.htm el 12 de marzo 2008.

Beneke, S., 2009. Diagrama sobre programas para niños pequeños. Sistema Conceptual para Illinois, 11(1). Descargado de http://ecrp.uiuc.edu/v1 Inl/beneke-sp.html el 13 de abril 2012. 
Bradley, RH, D. Vandell, 2007. Child care ant the well-being of children. Arch. Pediatr. Adolesc. Med., 16(7), 669-76. Descargado de http://www.ncbi.nlm.nih.gov/pubmed/17606830 en agosto 2012.

Byington, T.A., M.T. Tannock, 2011. Los intereses y necesidades del desarrollo profesional para instructores en el ámbito de la educación infantil. ECRP Early Child Research and Practice, 13(2). Descargado de http://ecrp.uiuc.edu/v13n2/byington.html en junio 2012.

CNNA, 2009. Agenda Social de la Niñez y Adolescencia 2007-2010. Concejo Nacional de la Niñez y Adolescencia (CNNA), Quito, Ecuador.

Consejo Nacional de Discapacidades. (s.f.). La discapacidad en cifras. Descargado de http://www.conadis.gob.ec/docs/proyectoinversionderechos10.pdf en agosto 2012.

CCNNA, 2007. Agenda Social de la Niñez y Adolescencia 2007-2010. Consejo Nacional de la Niñez y Adolescencia (CCNN), Quito, Ecuador, 28 pp.

Douglas, A., 2011. Mejoras en la participación familiar. El contexto organizacional y la influencia en la colaboración con familias en programs formales de cuidado infantil. ECRP Early Child Research and Practice, 13(2). Descargado de http://ecrp.uiuc.edu/v13n2/douglass.html el 13 de abril 2012.

Educiudadania, 2011. Acompañando al Plan Decenal de Educación. Organización coordinadora Grupo Faro, Quito, Ecuador, 6-9.

FEAPS, 1999. Atención Temprana: Orientaciones para la Calidad. Confederación española de Organizaciones a Favor de las Personas con Retraso Mental (FEAPS), Madrid, España.

Goldstein, J., D.B. McCoach, 2011. Punto de partida. El desarrollo de una estructura para la evaluación por maestros de las habilidades de estudiantes a la entrada al kindergaten. ECRP: Investigación y Práctica de la Niñez Temprana, 13(2). Descargado de http://ecrp.uiuc.edu/v13n2/goldstein-sp.html el 10 de abril 2012.

Huiracocha, L., G. Robalino, M.S. Huiracocha, J.L. García, C.G. Pazán, A. Angulo, 2012. Retrasos del desarrollo psicomotriz en niños y niñas urbanos de 0 a 5 años: Estudio de caso en la zona urbana de Cuenca, Ecuador. Maskana, 3(1), 13-28.

INNFA, 2007. Currículo Intermedio de Educación Inicial. Instituto Nacional de la Niñez y la Familia (INNFA), Quito, Ecuador, 80 pp.

McWilliams, C., S.C. Ball, S.E. Benjamin, D. Hales, A. Vaughn, D.S. Ward, 2009. Best practice guidelines for physical activity at child care. Pediatrics, 124(6), 1650-9. Michigan Department of Education, 2006. Early childhood standards of quality for infant and toddler programas. Michigan Department of Education, Michigan, USA, 99 pp.

Ministerio de Educación, 2012. Desarrollo Profesional Educativo. Educación Inicial [En línea]. Descargado de http://www.educacion.gob.ec/index.php/educacion-inicial-pdle el 15 de enero 2012.

Ortiz, N. , 1999. Escala Abreviada de Desarrollo. UNICEF, Colombia, 61 pp.

Oberhuermer, 2000. Conceptualizing the professional role in Early Childhood Centers. ECRP Early Child Research Practice,2(2). Descargado de http://ecrp.uiuc.edu/bytitle.html el 14 de abril 2012.

Observatorio de la Discapacidad, 2004. Guía de estándares de calidad en atención temprana. Programa INTER-IMSERSO, Madrid, España, 45 pp.

Peñafiel, F., A. Hernández, A. Chacón, 2003. Atención Temprana. Enseñanza, 21, 245- 274.

Raina, P., M. Donnell, P. Rosenbaum, J. Brehaut, S.D. Walter, M. Swinton, B. Zhu, E. Wood, 2005. The health and well-being of caregivers of children with cerebral palsy. Pediatrics, 115(6); 62636.

Rezende, M., M. Andrade, V. Beteli, J. Santos, 2005. Follow up of the child's motor abilities in day care center and pre-schools. Rev. Latino-Am. Enfermagem [on line], 13(5), 619-625.

Rosero, J., 2009. Estado Situacional de los niños y niñas en los Programas Públicos de Desarrollo Infantil del Ecuador. Ministerio de Coordinación de Desarrollo Social, Quito, Ecuador, 14-16. 
Scott, C., J. Lesko, J. Martella, 2007. Las pautas prescolares: resultados de una encuesta nacional para documentar tendencias en las políticas y practicas a nivel estatal. Descargado de http://ecrp.uiuc.edu/v9n1/little-sp.html el 12 de abril 2009.

Somers, T.S., H.M. Somers, T.S. Harvey, M.L. Rusnak, 2011. Making child centers safer: a non regulatory approach to improving child care center. Public Health Rep., 126(1), 34-40.

UNICEF, 2006. Estado de los derechos de la Niñez y la Adolescencia en el Ecuador 2005. Fundación Observatorio Social del Ecuador, UNICEF, Ecuador, 101-104.

Walsh, G., J. Gardner, 2005. Evaluaciones de la calidad de ambientes para los primeros años de aprendizaje. ECRP Early Child Research Practice, 7(1). Descargado de http://ecrp.uiuc.edu/v7n1/walsh-sp.html el 5 de junio 2008. 